\title{
Lead in bottled waters: contamination from glass and comparison with pristine groundwater
}

\author{
William Shotyk* and Michael Krachler \\ Institute of Environmental Geochemistry, University of Heidelberg, \\ INF 236, D-69120 Heidelberg, GERMANY \\ email: shotyk@ugc.uni-heidelberg.de \\ tel +49 (6221) 544803 fax 545228
}

*corresponding author

revised version, re-submitted for publication in Environmental Science \& Technology

word count: 4691 plus Two Tables and Three Figures $(1500)=6191$

Electronic Supplementary Information: One Table S1 and One Figure S1a and S1b 


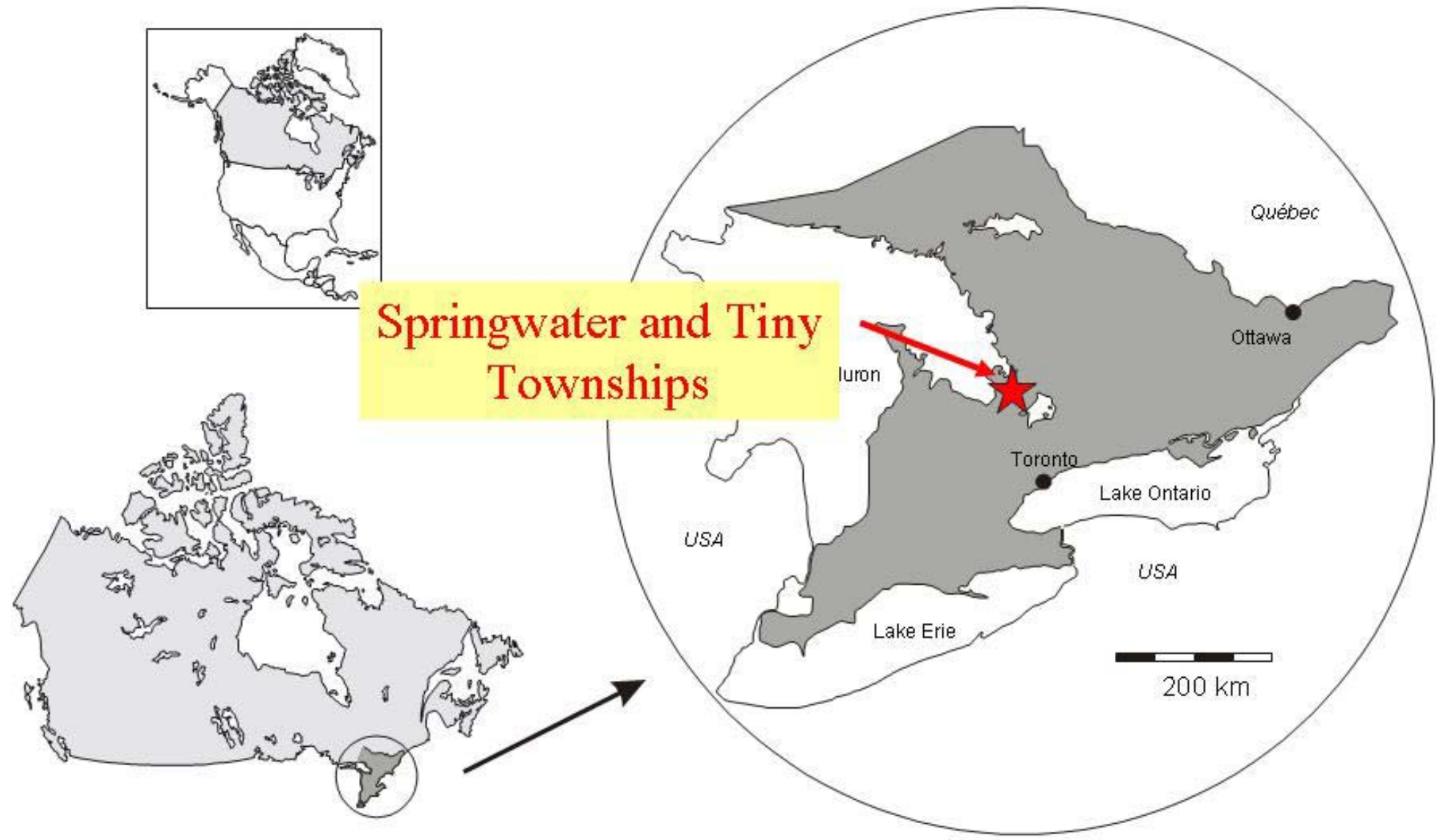

Figure S1a, Supplementary Information 


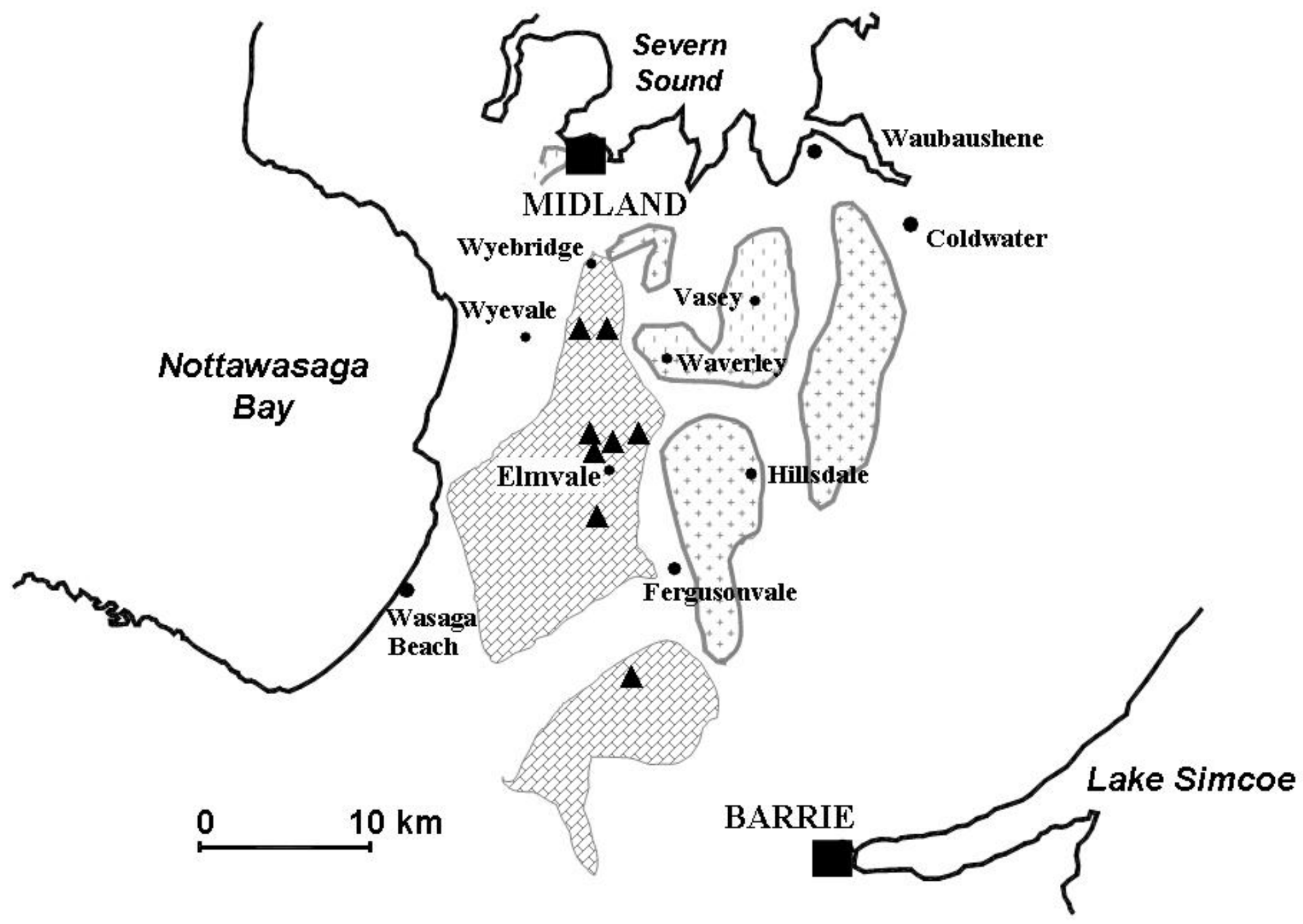

Figure S1b, Supplementary Information 
Table S1 Supplementary Information Lead concentrations (ng/l), container material, type of water and country of origin of all bottled waters investigated.

\begin{tabular}{|c|c|c|c|c|}
\hline bottle ID \# & Pb, ng/l & $\begin{array}{l}\text { container } \\
\text { materiall }^{\mathrm{c}}\end{array}$ & type of water & country \\
\hline 1a & 56 & PET & natural & Australia \\
\hline $1 b$ & 54 & PET & natural & Australia \\
\hline $2 \mathrm{a}$ & 35 & PET & natural & Australia \\
\hline $2 b$ & 35 & PET & natural & Australia \\
\hline 3a & 411 & PET & natural & Australia \\
\hline $3 b$ & 251 & PET & natural & Australia \\
\hline $4 a$ & 268 & PET & natural & Australia \\
\hline $4 \mathrm{~b}$ & 307 & PET & natural & Australia \\
\hline $5 a$ & 6,6 & PET & natural & Belgium \\
\hline $5 b$ & 8,4 & PET & natural & Belgium \\
\hline $5 c$ & 9,7 & PET & natural & Belgium \\
\hline $6 a$ & 246 & PET & natural & Brazil \\
\hline $6 b$ & 249 & PET & natural & Brazil \\
\hline $7 \mathrm{a}$ & 46 & PET & natural & Brazil \\
\hline $7 b$ & 32 & PET & natural & Brazil \\
\hline $8 a$ & 3,0 & $\mathrm{PP}$ & natural & Canada \\
\hline $8 b$ & 3,4 & PP & natural & Canada \\
\hline $8 c$ & 4,2 & PP & natural & Canada \\
\hline $8 d$ & 2,5 & PP & natural & Canada \\
\hline $8 \mathrm{e}$ & 2,4 & PP & natural & Canada \\
\hline $8 f$ & 3,2 & PP & natural & Canada \\
\hline $8 g$ & 3,8 & PP & natural & Canada \\
\hline $8 \mathrm{~h}$ & 4,6 & $\mathrm{PP}$ & natural & Canada \\
\hline 9 & 6,1 & PET & tap & Canada \\
\hline 10a & 2,8 & PET & natural & Canada \\
\hline 10b & 7,9 & PET & natural & Canada \\
\hline $10 \mathrm{c}$ & 7,3 & PET & natural & Canada \\
\hline 11 & 23 & PET & natural & Canada \\
\hline 12 & 4,1 & PET & natural & Canada \\
\hline 13 & 26 & PET & natural & Canada \\
\hline 14 & 2,1 & PET & natural & Canada \\
\hline $15 a^{b}$ & 159 & PET & natural & Canada \\
\hline $15 b$ & 154 & PET & natural & Canada \\
\hline $15 c$ & 155 & PET & natural & Canada \\
\hline $15 d$ & 5,7 & PET & natural & Canada \\
\hline $15 \mathrm{e}$ & 10 & PET & natural & Canada \\
\hline $15 f$ & 42 & PET & natural & Canada \\
\hline 16 & 268 & PET & natural & Canada \\
\hline 17 & 1,6 & PET & tap & Canada \\
\hline 18 & 47 & PET & natural & Canada \\
\hline
\end{tabular}




\begin{tabular}{|c|c|c|c|c|}
\hline $19 a$ & 17 & PET & natural & Canada \\
\hline $19 b$ & 23 & PET & natural & Canada \\
\hline $19 c$ & 32 & PET & natural & Canada \\
\hline 19d & 36 & PET & natural & Canada \\
\hline $19 \mathrm{e}$ & 3,8 & PET & natural & Canada \\
\hline 20 & 27 & PET & natural & Canada \\
\hline 21 & 35 & PET & natural & Canada \\
\hline 22 & 5,5 & PET & natural & Canada \\
\hline 23 & 2,6 & PET & natural & Canada \\
\hline 24 & 4,6 & PET & natural & Canada \\
\hline 25 & 4,2 & PET & natural & Canada \\
\hline 26 & 18 & PET & natural & Canada \\
\hline 27 & 3,0 & PET & tap & Canada \\
\hline $28 \mathrm{a}$ & 25 & Other & tap & Canada \\
\hline $28 b$ & 34 & Other & tap & Canada \\
\hline $28 c$ & 48 & Other & tap & Canada \\
\hline $28 d$ & 59 & PET & tap & Canada \\
\hline 29 & 13 & PET & natural & Canada \\
\hline 30 & 40 & PET & tap & Canada \\
\hline 31 & 11 & PET & tap & Canada \\
\hline 32 & 143 & PET & natural & Canada \\
\hline 33 & 8,4 & PET & natural & Czech Republic \\
\hline 34 & 27 & PET & natural & Czech Republic \\
\hline 35 & 5,6 & PET & natural & Czech Republic \\
\hline 36 & 13 & PET & natural & Czech Republic \\
\hline 37 & 5,3 & PET & natural & Denmark \\
\hline 38 & 2,4 & PET & natural & Denmark \\
\hline 39 & 3,2 & PET & natural & Denmark \\
\hline 40 & 72 & PET & natural & Dominican Republic \\
\hline 41 & 4,1 & PET & tap & Dominican Republic \\
\hline 42 & 145 & PET & natural & Dominican Republic \\
\hline 43 & 3,2 & PET & natural & England \\
\hline 44 & 2,2 & PET & natural & England \\
\hline 45 & 47 & PET & natural & Finland \\
\hline 46 & 16 & PET & natural & Finland \\
\hline 47 & 38 & PET & natural & Finland \\
\hline 48 & 48 & PET & natural & Finland \\
\hline $49 a$ & 7,6 & PET & natural & France \\
\hline $49 b$ & 8,0 & PET & natural & France \\
\hline $50 \mathrm{a}$ & 6,7 & PET & natural & France \\
\hline $50 \mathrm{~b}$ & 5,1 & PET & natural & France \\
\hline 51 & 7,1 & PET & natural & France \\
\hline 52 & 7,2 & PET & natural & France \\
\hline 53 & 14 & PET & natural & France \\
\hline 54 & 2,1 & PET & natural & France \\
\hline 55 & 9,9 & PET & natural & France \\
\hline 56 & 15 & PET & natural & France \\
\hline
\end{tabular}




\begin{tabular}{|c|c|c|c|c|}
\hline 57 & 4,6 & PET & natural & France \\
\hline 58 & 2,6 & PET & natural & France \\
\hline $59 a$ & 12 & PET & natural & Germany \\
\hline $59 b$ & 13 & PET & natural & Germany \\
\hline $59 c$ & 9,7 & PET & natural & Germany \\
\hline 59d & 18 & PET & natural & Germany \\
\hline 59e & 8,8 & PET & natural & Germany \\
\hline $59 f$ & 22 & PET & natural & Germany \\
\hline $60 a$ & 99 & PET & natural & Germany \\
\hline $60 \mathrm{~b}$ & 9,9 & PET & natural & Germany \\
\hline 60c & 5,4 & PET & natural & Germany \\
\hline $60 d$ & 4,9 & PET & natural & Germany \\
\hline $60 \mathrm{e}$ & 5,5 & PET & natural & Germany \\
\hline $60 f$ & 3,6 & PET & natural & Germany \\
\hline $61 \mathrm{a}$ & 23 & PET & natural & Germany \\
\hline $61 b$ & 84 & PET & natural & Germany \\
\hline 61c & 11 & PET & natural & Germany \\
\hline 61d & 7,8 & PET & natural & Germany \\
\hline $61 \mathrm{e}$ & 57 & PET & natural & Germany \\
\hline $61 f$ & 8,8 & PET & natural & Germany \\
\hline 62 & 11 & PET & natural & Germany \\
\hline 63 & 37 & PET & natural & Germany \\
\hline 64 & 99 & PET & natural & Germany \\
\hline 65 & 23 & PET & natural & Germany \\
\hline 66 & 14 & PET & natural & Germany \\
\hline 67 & 6,5 & PET & natural & Germany \\
\hline 68 & 7,1 & PET & natural & Germany \\
\hline 69 & 5,7 & PET & natural & Germany \\
\hline 70 & 3,9 & PET & natural & Germany \\
\hline 71 & 16 & PET & natural & Germany \\
\hline $72 a$ & 4,2 & PET & natural & Germany \\
\hline $72 b$ & 5,1 & PET & natural & Germany \\
\hline $72 c$ & 6,6 & PET & natural & Germany \\
\hline $73 a$ & 105 & PET & tap & Germany \\
\hline $73 b$ & 138 & PET & tap & Germany \\
\hline $73 c$ & 113 & PET & tap & Germany \\
\hline 74 & 20 & PET & natural & Hong Kong \\
\hline 75 & 8,1 & PET & tap & Hong Kong \\
\hline 76 & 31 & PET & natural & Hong Kong \\
\hline 77 & 5,6 & PET & natural & Hong Kong \\
\hline 78 & 2,3 & PET & natural & Hong Kong \\
\hline 79 & 10 & PET & natural & Iceland \\
\hline $80 a$ & 3,5 & PET & natural & Israel \\
\hline $80 \mathrm{~b}$ & 3,3 & PET & natural & Israel \\
\hline $80 c$ & 5,5 & PET & natural & Israel \\
\hline $81 a$ & 7,4 & PET & natural & Israel \\
\hline
\end{tabular}




\begin{tabular}{|c|c|c|c|c|}
\hline $81 b$ & 6,6 & PET & natural & Israel \\
\hline 81c & 7,8 & PET & natural & Israel \\
\hline 82 & 8,3 & PET & natural & Italy \\
\hline 83 & 10 & PET & natural & Italy \\
\hline 84 & 5,6 & PET & natural & Japan \\
\hline 85 & 5,1 & PET & natural & Japan \\
\hline 86 & 3,2 & PET & natural & Japan \\
\hline 87 & 13 & PET & natural & Japan \\
\hline 88 & 63 & PET & natural & Japan \\
\hline 89 & 7,3 & PET & natural & Japan \\
\hline 90 & 4,0 & PET & natural & Japan \\
\hline 91 & 4,2 & PET & natural & Japan \\
\hline 92 & 2,8 & PET & natural & Japan \\
\hline 93 & 3,3 & PET & natural & Japan \\
\hline 94 & 17 & PET & natural & Japan \\
\hline 95 & 2,2 & PET & natural & Japan \\
\hline 96 & 203 & PET & natural & Kenya \\
\hline $97 a$ & 4,0 & PET & tap & Mexico \\
\hline $97 \mathrm{~b}$ & 3,3 & PET & tap & Mexico \\
\hline 98 & 2,4 & PET & tap & Mexico \\
\hline 99 & 4,4 & PET & tap & Mexico \\
\hline 100 & 760 & PET & natural & Peru \\
\hline 101 & 40 & PET & natural & Poland \\
\hline 102 & 15 & PET & natural & Poland \\
\hline 103 & 11 & PET & natural & Slovenia \\
\hline 104 & 6,9 & PET & natural & Spain \\
\hline 105 & 29 & PET & tap & Spain \\
\hline 106 & 56 & PET & natural & Spain \\
\hline 107 & $<1$ & PET & natural & Spain \\
\hline 108 & 6,4 & PET & natural & Spain \\
\hline 109 & 75 & PET & natural & Spain \\
\hline 110 & 5,5 & PET & natural & Spain \\
\hline 111 & 2,5 & PET & natural & Sweden \\
\hline 112 & 41 & PET & natural & Sweden \\
\hline 113 & 8,5 & PET & natural & Switzerland \\
\hline 114 & 3,2 & PET & natural & Switzerland \\
\hline 115 & 8,6 & PET & natural & Switzerland \\
\hline 116 & 13 & PET & natural & Switzerland \\
\hline 117 & 20 & PET & natural & The Netherlands \\
\hline 118 & 3,2 & PET & natural & The Netherlands \\
\hline 119 & $<1$ & PET & tap & Trinidad \\
\hline 120 & 5,5 & PET & tap & USA \\
\hline 121 & 4,9 & PET & tap & USA \\
\hline 122 & 13 & PET & tap & USA \\
\hline 123 & 4,4 & PET & natural & USA \\
\hline $124 a$ & 18 & BP & natural & USA \\
\hline $124 b$ & 5,0 & $\mathrm{BP}$ & natural & USA \\
\hline
\end{tabular}


natural

USA

$124 d$

4,1 BP

natural

USA

$124 \mathrm{e}$

2,9 BP

natural

USA

$124 \mathrm{f}$

3,2

BP

natural

USA

125

24

PET

natural

Yugoslavia

${ }^{a}$ numbers refer to the brand of bottled water and letters indicate replicates of the corresponding brand.

${ }^{\mathrm{b}}$ bottles 15a-c and 15d-f were purchased in the summers of 2005 and 2003, respectively.

c "PET” Polyethylene terephthalate, "PP” Polypropylene; "BP” labeled "Bioplastic" “Other” refers to plastics other than PET, PP, high or low density PE (polyethylene), PVC

(polyvinylchloride) or PS (polystyrene), and maybe a mixture of several kinds of plastic. 